\title{
La programación por competencias básicas: hacia un cambio metodológico interdisciplinar
}

\section{Programming through the key competencies: towards an interdisciplinary methodological change}

\author{
Beatriz SIERRA Y ARIZMENDIARRIETA, Antonio MÉNDEZ-GIMÉNEZ \\ y Jorge MAÑANA-RODRÍGUEZ \\ Universidad de Oviedo y Consejo Superior de Investigaciones Científicas (CSIC)
}

\section{Recibido: Marzo 2012}

Aceptado: Junio 2012

\section{Resumen}

La inclusión de las competencias en el Curriculum está generando ciertos problemas, tanto en las programaciones como en su desarrollo en el aula. Su incorporación no ha de entenderse como si fuera un elemento más, sino que precisamente el cambio conceptual y metodológico viene de la mano de la consideración de las competencias como el eje en torno al cual han de girar todos y cada uno de los elementos curriculares. En este trabajo realizamos una propuesta de carácter metodológico-prospectivo en la que defendemos que dicho cambio en la planificación debe plantearse en dos niveles: dentro y fuera del aula, a través del trabajo docente cooperativo e interdisciplinar.

Palabras clave: Competencias, programación, innovación, metodología interdisciplinar.

\begin{abstract}
The inclusion of the competences in the Curriculum is generating certain problems, in the programming as well as in its development in the classroom. Its inclusion should not be understood as just another element, but, precisely, the conceptual and methodological change comes from the consideration of the competences as the axis around which all curricular elements must spin. In this paper, a methodological-prospective proposal is shown according to which this change in the process will have to be planned in two levels: inside and outside the classroom, through cooperative and interdisciplinary teachers' work.
\end{abstract}

Keywords: Competences, programming, innovation, cross curricular methodology.

La innovación que supone la enseñanza basada en competencias no puede llevarse a cabo con las programaciones de siempre. Añadir las competencias a lo que ya teníamos no cambia nada. Si de algo sirve una enseñanza que pretenda desarrollar las ocho 
competencias básicas (tanto en primaria como en ESO) es precisamente para dotar de sentido a contenidos que a veces se han planteado de forma separada del contexto vital de los estudiantes. Las competencias básicas pueden resultar una herramienta eficaz (pues no son fin, sino medio) para que el alumnado sea capaz de aprender de manera más viva y contextualizada pudiendo entender y desarrollar su creatividad a partir de problemas que les permitan comprender de forma significativa los diversos contenidos. Pero para que esto sea posible el profesorado ha de replantearse su tarea en un doble sentido: tanto en su planificación docente como en su desarrollo en el aula.

Estas páginas tienen básicamente dos objetivos:

1. Responder a la pregunta acerca del sentido o lugar de las competencias en el currículo y las exigencias curriculares que se derivan de su inclusión en el mismo.

2. Como consecuencia de lo anterior, reflexionar acerca del cambio metodológico que plantea la enseñanza basada en competencias si quiere ser realmente eficaz.

\section{Un modo de plantear las nuevas programaciones por competencias}

La inclusión de las competencias básicas en el currículo exige la adopción de una nueva perspectiva para su diseño y desarrollo. La introducción de las competencias básicas implica que éstas se constituyan en el eje que da sentido y articula el resto de los elementos curriculares. Lo que, a efectos prácticos, ha de llevar a concebir las programaciones desde un nuevo enfoque y no a incorporar simplemente un apartado más. La inclusión de las competencias básicas no debe realizarse como un añadido a lo que ya había, pues de ser así no supondrían ningún cambio. La idea de un aprendizaje basado en competencias supone que éstas han de constituir el hilo conductor en torno al cual se configura el resto de los elementos del currículo. De modo que las competencias, o mejor, los diferentes aspectos o capacidades a desarrollar de cada una de las competencias deben estar presentes y ligados en cada momento tanto a objetivos, contenidos y actividades, y evaluación, mediante una metodología más interdisciplinar y globalizada. Todos estos elementos curriculares han de sufrir una modificación no sólo a nivel organizativo formal, sino estructural, por cuanto se transforma el planteamiento mismo de los procesos de enseñanza-aprendizaje. Jurado propone superar las perspectivas academicista y operacionalista -en las que el aprendiz repite la información o hace las cosas tal y como se le ha enseñado- con una perspectiva multidimensional, que "supone romper con los compartimentos curriculares para apuntar hacia la transversalidad" (Jurado Valencia, 2009, pp. 347), de manera que el alumnado se pregunte constantemente por el sentido de lo que aprende, trascendiendo los espacios y tiempos escolares.

Se puede enseñar a hacer una suma, pero no a ser competente en matemáticas, pues ello implica el desarrollo de diferentes aspectos de la competencia matemática con el ejercicio en contextos diversos. Si ya hace años se insistía en la actividad y participación del alumnado en su propio aprendizaje, recalcando que no había de ser un mero receptor pasivo de informaciones, ahora más que nunca se hace patente dicha 
actividad. Con el modelo actual, que el aprendiz sea activo no es sólo una intención del docente, sino que se hace imprescindible como una estrategia metodológica ineludible en la enseñanza por competencias. Se puede decir que no se puede enseñar competencias de modo directo, sino que se trata de promover el desarrollo de las mismas en el alumnado, que habrá de adquirirlas con la práctica y la ayuda docente. "A la escuela le compromete, afirma Jurado, propiciar condiciones para que sus participantes (estudiantes, docentes, directivos y padres) vivencien el asombro de conocer y construyan la actitud ética y volitiva que ello implica" (Jurado Valencia, 2009, pp. 348-349).

En sentido estricto, no se enseñan o aprenden competencias, sino que se desarrollan capacidades que hacen, a quien realiza determinadas tareas, ser más competente. La adquisición de competencias es, por ello, gradual, teniendo como consecuencia que la evaluación de una competencia siempre resulta parcial y nunca directamente evaluable. Lo que se puede evaluar son aspectos en los que se puede desglosar (de una forma más general o concreta) una competencia y no ésta directamente. Precisamente por este motivo, a la hora de programar será preciso detallar diferentes aspectos en los que se va a trabajar las distintas competencias y no simplemente mencionar la competencia de modo general. Oliveros distingue entre el "desarrollo de competencias", asociado a la formación en el puesto de trabajo, y "la formación en competencias", ligada a la enseñanza en el aula, precisamente a partir de que en la última no se ejercita la competencia en su totalidad, sino aspectos o componentes de la misma (Oliveros Martín-Vares, 2006, p. 107). En el aprendizaje de las competencias, como ya hemos dicho, lo que se aprende en realidad no es la competencia, sino el saber realizar diversas acciones que integradas y puestas en práctica en diversos contextos, configuran una competencia. La evaluación de competencias, por tanto, no se realiza sobre los contenidos aprendidos, sino sobre la capacidad para utilizar los aprendizajes en distintas situaciones. De ahí que evaluar competencias sea "evaluar sistemas de reflexión y acción" (Viso Alonso, 2010, p. 148).

Consideramos, por tanto, que a la hora de programar es preciso disponer de unos listados de aspectos a trabajar de cada una de las competencias, bien sean los que aquí tomaremos como referencia -como más adelante indicaremos- u otros que cada docente pueda elaborar. Siempre teniendo en cuenta que, para dotar a la programación de cierta sistematicidad y rigor -huyendo de planteamientos arbitrarios y/o puntuales-, se ha de poder justificar la procedencia de los mismos.

A continuación, ofrecemos un esquema de cómo ubicar las competencias en las programaciones y algunas indicaciones para el desarrollo de cada apartado de las mismas. No hay una única manera de programar y cada docente habrá de encontrar la forma que se adapte mejor a sus necesidades. Por eso únicamente daremos unas pistas de cómo podría abordarse una programación incluyendo diferentes aspectos de cada una de las competencias. 


\section{Esquema de programación}

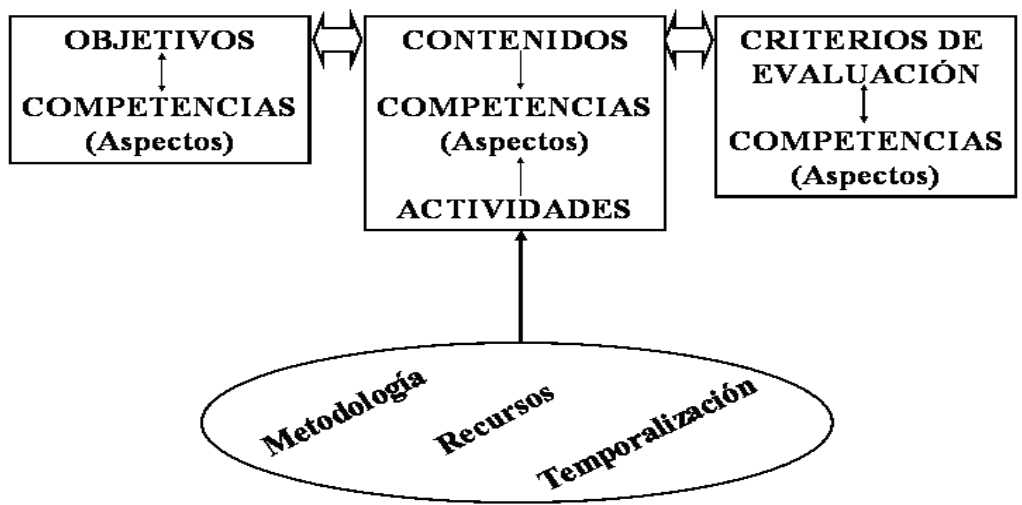

Figura 1: Las competencias básicas en el currículo Fuente: Elaboración propia

Las competencias han de estar en íntima relación con los objetivos, contenidos, actividades y evaluación. Por ello, es lógico pensar que para que exista una coherencia interna, tanto en lo planteado en la programación como en su implementación y desarrollo real en el aula, se han de tener muy claros los aspectos que se quieren trabajar en la unidad de tiempo para la que se programa. De otro modo, se corre el riesgo de que a la hora de plantear los objetivos estemos pensando en unos determinados aspectos de cada competencia y después no se desarrollen en las actividades y tareas planteadas, o no nos acordemos de ellos en la evaluación. Así, en cada programación sería conveniente fijar al comienzo los aspectos que se quieren desarrollar para cada competencia, siendo conscientes de que dichos aspectos condicionarán el modo de plantear toda la programación. Las nuevas programaciones deberían incluir, por tanto, los siguientes apartados:

1. Aspectos a desarrollar de cada competencia. Se entenderá que, en adelante, siempre que en los siguientes apartados de la programación se mencione una determinada competencia, nos estaremos refiriendo a los aspectos señalados al comienzo de la misma. Esto evita las repeticiones y, además, hace explícita la necesidad de intentar trabajar cada competencia gradualmente, atendiendo a los aspectos mencionados en cada unidad didáctica o en los periodos de tiempo en los que se divida la PGA, y evitando dispersiones o planteamientos más o menos difusos.

2. Objetivos y competencias. Dado el planteamiento interdisciplinar que subyace al desarrollo de las competencias, cada objetivo se relacionará con más de una competencia, debiendo estar las ocho presentes en el conjunto de los objetivos. La competencia afín a la materia que se programe se encontrará, 
como es lógico, presente en todos los objetivos. Y las otras siete competencias se asociarán al resto de los objetivos en función de su relación.

3. Contenidos y competencias. El concepto de competencia asume e integra tanto el 'saber' como el 'saber hacer', por lo que, en principio, no tendría por qué ser necesario desglosar los contenidos en conceptuales, procedimentales y actitudinales, aunque tampoco lo impediría.

4. Actividades y competencias. La forma de plantear las actividades ha de ser más integradora para poder articular contenidos de varias materias a través de la resolución de problemas en diferentes situaciones o contextos, bien sean reales o simulados. Este planteamiento ayuda a perfilar mejor la distinción entre actividades y ejercicios y tareas, frontera muy difusa a lo largo de los últimos años (Feito Alonso, 2010). Los ejercicios suelen emplearse más bien para el logro de determinadas destrezas a través de la repetición, sin tener en cuenta contextos, ni una necesaria relación entre unos y otros. Las actividades de las que hablamos tienen que tener un carácter más abarcante, pudiendo hablar de macroactividades a partir de las cuales se organicen diferentes tareas. Algunos autores destacan la importancia que cobran las tareas con la enseñanza por competencias (Domingo Segovia y Barrero Fernández, 2010; Rodríguez Torres, 2010; Viso Alonso, 2010). Las tareas pueden plantearse interrelacionadas de manera que cobren sentido a través de la macroactividad de la que forman parte. En cada una de ellas pueden trabajarse diferentes competencias. Si se quiere que el alumnado desarrolle la creatividad y la imaginación para resolver problemas nuevos, no podemos plantear las actividades de siempre y 'añadirles' competencias. Puede resultar interesante incorporar actividades no convencionales (Marco Stiefel, 2008, pp. 52-59). Estamos de acuerdo con Moya Otero cuando afirma que la estructura de tareas, y el contexto en el que se desarrollan, se convierte en el centro de atención. "Las tareas -escribe- configuran situaciones-problema que cada alumno debe tratar de resolver haciendo un uso adecuado de los contenidos escolares", de manera que son "microcontextos de aprendizaje" (Moya Otero, 2008, p. 73). Esta forma de abordar las actividades tiene, obviamente, una clara vinculación con un cambio metodológico y la utilización de diferentes recursos. Un ejemplo de metodología que puede enlazar perfectamente con este planteamiento es el Método de Proyectos (Arreaza Beberide, 2009; Jurado Valencia, 2009; Rosales López, 2010), aunque lo adecuado sería combinar diferentes métodos adaptados a cada nuevo contexto o situación.

5. Temporalización, metodología y recursos. Estos tres elementos sufren obviamente un cambio importante con la incorporación de las competencias. Lógicamente estarán los tres ligados y exigirán a partir de este momento mayor flexibilidad. Al tener que articular y trabajar todas las competencias desde cada una de las materias, se facilita una mayor conexión también entre los diferentes contenidos, lo que favorece metodologías y recursos más interdisciplinares. Del mismo modo, tanto los espacios como los tiempos tendrán que dejar de ser tan rígidos y adaptarse a métodos más globalizados que necesitarán espacios 
diversos y tiempos flexibles. No será ya el tiempo lo que condicionará los contenidos a trabajar o la actividad a realizar, sino que serán las actividades y tareas propuestas las que tendrán que marcar tiempos y horarios, y las distintas interacciones de contenidos y docentes, pues "en la aplicación de la tarea deben intervenir el mayor número de disciplinas posible" (García Peinado, 2009, p. 2).

6. Criterios de evaluación y competencias. Recordemos que en el Real Decreto de Enseñanzas Mínimas (Primaria y ESO) se afirma que "los criterios de evaluación, además de permitir la valoración del tipo y grado de aprendizaje adquirido, se convierten en referente fundamental para valorar el desarrollo de las competencias básicas". Las competencias no se identifican con los objetivos, ni con los criterios de evaluación, pero constituyen el referente último a cuya luz cobran sentido ambos. En realidad, los objetivos y los criterios de evaluación son dos caras de la misma moneda. Los objetivos proyectan hacia el futuro lo que queremos conseguir en el aprendizaje del alumnado, un desideratum, y podríamos definir los criterios de evaluación como la expresión de los mismos objetivos, pero a modo de comprobación o valoración presente de la evolución o finalización de un proceso. En definitiva, los criterios de evaluación pretenden asir lo que han dado de sí nuestros primeros deseos. Pretenden valorar, o medir, la realización concreta de las posibilidades, aún abstractas por no haberse cumplido, que expresan esperanzadamente los objetivos. En este sentido, en el momento actual cobran especial interés los 'indicadores de desempeño', que actúan concretando y contextualizando los criterios de evaluación. Asumimos la definición ofrecida por Escamilla al definirlos como: "Enunciados que, respecto a una o varias competencias dadas, identifican un tipo de guía o patrón de conducta adecuado, eficaz, positivo (suponen siempre evolución y desarrollo). Proporcionan, al tiempo, una vía directa para determinar, de manera objetivable, el grado (cuantitativo o cualitativo) en que se manifiestan" (Escamilla, 2009, p. 209).

Hasta la incorporación de las competencias, en la programación se relacionaban únicamente los criterios de evaluación con los objetivos planteados para comprobar el grado de acuerdo entre ellos y permitir la evaluación de los contenidos aprendidos. En las nuevas programaciones habremos de evaluar tanto el logro o grado de adquisición de los objetivos como de las competencias desarrolladas. Según ya hemos avanzado, no se puede evaluar una competencia de modo general y, por tanto, desglosar (por el procedimiento que sea) cada competencia resulta extremadamente útil. Para poder evaluar no sólo los objetivos, sino el grado de adquisición de los aspectos a desarrollar de cada competencia, plasmados en el primer apartado, habrá que tomar como punto de referencia los criterios de evaluación del ciclo correspondiente para cada una de las áreas, de manera que se puedan evaluar todas las competencias (si se trata de las cuatro competencias con mayor afinidad con un área). Para las competencias de carácter más trasversal, encontramos referentes de evaluación en todas las áreas. 
Por no ser el objetivo de nuestro artículo, no entraremos en la discusión teóricopráctica acerca de la evaluación de las competencias, tan candente en la bibliografía del momento, aunque sí queremos apuntar el problema de la elección de una evaluación más cuantitativa o cualitativa. La dificultad de la evaluación de competencias que requieren un conocimiento más reflexivo no se resuelve fácilmente con pruebas objetivas que pretendan medir resultados, o evaluar "sólo con tareas de lápiz y papel" (Monereo y Pozo, 2007, p. 18). La solución más difícil, sin duda, pero más coherente con este planteamiento, tiene que ver más con la valoración de resultados que con su medida. Aún así, y seguramente por la dificultad que entraña, se producen deslizamientos a un planteamiento evaluativo que pretende medir objetiva y cuantitativamente competencias reflexivas, convirtiéndolas así -consciente o inconscientemente- en competencias técnicas. Rosales (2010) distingue entre evaluación de procesos y evaluación final. En este sentido, puede verse, entre otras, las reflexiones de Zabala y Arnau (2007) o Álvarez Méndez (2009). Resulta también enriquecedor, para analizar la autenticidad de una tarea de evaluación, la propuesta del término "proximidad ecológica" que "se refiere a la conexión de la modalidad de evaluación elegida con las prácticas habituales del profesorado que las pone en juego, dentro de su zona de desarrollo docente" (Monereo, 2009, p. 14).

A continuación, trataremos de ofrecer un ejemplo de lo que acabamos de explicar. Lo que sigue debe entenderse más bien como una programación marco, pues no está concebida para implementarse en un contexto real, dado que lo plasmado en ella son mínimos - con alto grado de generalidad-planteados en el Real Decreto de Enseñanzas Mínimas de la Educación Primaria para el primer ciclo. Lo que nos interesa, por tanto, no es ofrecer un ejemplo de una programación real, sino mostrar cómo las competencias han de vincularse, desde dentro, con objetivos, contenidos y criterios de evaluación (por ello tampoco se desciende a los detalles que aportan los indicadores de desempeño). Así, extraemos los objetivos (con algunos cambios) a partir de los fijados en el Anexo 2 del Decreto, para toda la etapa, en el área de lengua castellana y literatura, y de los planteados en el Curriculum de Primaria de nuestra Comunidad Autónoma. Los contenidos y criterios de evaluación se toman literalmente de los que establece el Decreto para el primer ciclo del área mencionada. En primer lugar, como hemos indicado páginas atrás, se seleccionarán aquellos aspectos de las competencias que nos interesa trabajar. En segundo lugar, mostraremos en un cuadro (Tabla 1) la relación interna entre objetivos, contenidos y criterios de evaluación con los aspectos de las competencias con ellos asociados. Seleccionamos los aspectos de cada competencia del listado propuesto por Sierra y Arizmendiarrieta, Méndez-Giménez y Mañana Rodríguez (2012) para programar por competencias de una forma más operativa. Dichos aspectos han sido fruto de la elaboración de un procedimiento desarrollado en el marco de la primera fase del Proyecto "Desarrollo de competencias básicas en primaria: hacia una metodología cooperativa interdisciplinar en la tarea docente" 1 .

${ }^{1}$ Este Proyecto ha estado financiado, desde junio de 2007 a diciembre de 2010, a través del Programa 2 "Innovación e Investigación Educativa y Planes de Calidad" al amparo del 


\section{Aspectos a desarrollar de cada competencia}

Seleccionamos un aspecto de cada competencia y cuatro de la competencia afín al área que se programa, a la que lógicamente habrá que darle una mayor importancia mientras se trabaje con ella.

1. Competencia matemática: contribuir al desarrollo de las capacidades cognitivas como la abstracción, simbolización, deducción y formalización, procurando que éstas se apliquen tanto en la vida cotidiana como en todas las materias curriculares.

\section{Competencia en comunicación lingüística:}

-Comprender y expresarse oralmente y por escrito de forma adecuada en los diferentes contextos de la actividad social y cultural, haciendo uso de los conocimientos sobre la lengua y las normas del uso lingüístico.

-Ampliar el vocabulario y fijar la ortografía para escribir y hablar de forma adecuada, coherente y correcta, y para comprender textos orales y escritos, iniciando la reflexión progresiva sobre el uso de la lengua, estableciendo relaciones entre los aspectos formales y los contextos e intenciones comunicativas a los que responden, para mejorar el uso personal del lenguaje.

-Leer con fluidez y entonación adecuadas, utilizando la lectura como fuente de placer y de enriquecimiento personal, y aproximarse a obras relevantes de la tradición literaria para desarrollar hábitos de lectura.

-Identificar aspectos fonéticos, de ritmo, acentuación y entonación, así como estructuras lingüísticas y aspectos léxicos de las lenguas y usarlos como elementos básicos de la comunicación.

3. Competencia en el conocimiento e interacción con el mundo físico: Interpretar, expresar y representar hechos, conceptos y procesos del medio natural, social y cultural, mediante códigos numéricos, gráficos, cartográficos y otros.

4. Competencia cultural y artística: Conocer y valorar diferentes manifestaciones artísticas del patrimonio cultural propio y de otros pueblos, colaborando en la conservación y renovación de las formas de expresión locales y estimando el enriquecimiento que supone el intercambio con personas de diferentes culturas que comparten un mismo entorno.

5. Autonomía e iniciativa personal: Desarrollar una relación de auto-confianza con la producción personal, respetando las creaciones propias y las de las otras personas y sabiendo recibir y expresar críticas y opiniones.

6. Competencia para aprender a aprender: Mantener una actitud de búsqueda personal y colectiva, articulando la percepción, la imaginación, la indagación y la sensibilidad y reflexionando a la hora de realizar y disfrutar de diferentes producciones.

Convenio de Colaboración suscrito entre la Consejería de Educación y Cultura del Principado de Asturias y la Universidad de Oviedo, para actividades educativas de 31 de mayo de 2002, B.O.P.A. de 3 de agosto de 2002 . 
7. Tratamiento de la información y competencia digital: Buscar, seleccionar, registrar y analizar la información, utilizando técnicas y estrategias diversas para acceder a ella según la fuente a la que se acuda y el soporte que se utilice (oral, impreso, audiovisual, digital o multimedia).

8. Competencia social y ciudadana: Valorar las lenguas en general como medio de comunicación y entendimiento entre personas de procedencias y culturas diversas y como herramienta de aprendizaje de distintos contenidos, mostrando una actitud receptiva y respetuosa hacia sus hablantes y su cultura, y apreciándolas como muestra de riqueza cultural.

\begin{tabular}{|c|c|c|}
\hline $\begin{array}{l}\text { Objetivos y } \\
\text { Competencias }\end{array}$ & $\begin{array}{l}\text { Contenidos y } \\
\text { Competencias }\end{array}$ & $\begin{array}{c}\text { Criterios de Evaluación y } \\
\text { Competencias }\end{array}$ \\
\hline $\begin{array}{l}\text { 1. Comprender y expresarse } \\
\text { oralmente y por escrito de } \\
\text { forma adecuada en los } \\
\text { diferentes contextos de la } \\
\text { actividad social y cultural. } 1 \text {, } \\
2,3,4,5,6,7,8 \text {. (Contenidos } \\
\text { bloque } 1 \text { ) }\end{array}$ & $\begin{array}{l}\text { Bloque 1. Escuchar, hablar y } \\
\text { conversar } \\
\text { 1. Comprensión y valoración } \\
\text { de textos orales sobre hechos y } \\
\text { acontecimientos próximos a la } \\
\text { experiencia infantil } \\
\text { procedentes de la radio y la } \\
\text { televisión. } \\
\text { 2. Participación y cooperación } \\
\text { en situaciones comunicativas } \\
\text { del aula, valorando y } \\
\text { respetando las normas que } \\
\text { rigen la interacción social. }\end{array}$ & $\begin{array}{l}\text { 1. Describir y representar la situación } \\
\text { de un objeto del espacio próximo, y de } \\
\text { un desplazamiento en relación a la } \\
\text { propia persona, o a otros puntos de } \\
\text { referencia utilizando los conceptos de } \\
\text { izquierda-derecha, delante-detrás, } \\
\text { arriba-abajo, cerca-lejos y próximo- } \\
\text { lejano. }(1,2,3,5,6) \\
2 . \text { Realizar interpretaciones } \\
\text { elementales de los datos presentados } \\
\text { en gráficas de barras. Formular y } \\
\text { resolver sencillos problemas en los } \\
\text { que intervenga la lectura de gráficos. } \\
\text { Clasificar los sucesos cotidianos como } \\
\text { posibles, imposibles o seguros. }(1,2, \\
5,6)\end{array}$ \\
\hline $\begin{array}{l}\text { 7. Leer con fluidez y } \\
\text { entonación adecuadas, } \\
\text { comprendiendo distintos tipos } \\
\text { de textos adaptados a la edad } \\
\text { y utilizando la lectura para } \\
\text { ampliar el vocabulario, fijar la } \\
\text { ortografía y adquirir las } \\
\text { estructuras básicas de la } \\
\text { lengua. 1, 2, 5, 6. (Contenidos } \\
\text { bloque 2). }\end{array}$ & $\begin{array}{l}\text { Bloque } 2 \text {. Leer y escribir } \\
\text { 3. Lectura comprensiva en voz } \\
\text { alta con entonación adecuada } \\
\text { de textos breves, haciendo } \\
\text { especial hincapié en la correcta } \\
\text { vocalización, el ritmo y las } \\
\text { pausas. } \\
\text { 4. Escritura de textos breves, } \\
\text { copiando o al dictado con } \\
\text { reproducción correcta de todos } \\
\text { los grafemas, cuidado de la } \\
\text { buena presentación y } \\
\text { corrección ortográfica. }\end{array}$ & $\begin{array}{l}\text { 3. Participar en las situaciones de } \\
\text { comunicación del aula, respetando las } \\
\text { normas del intercambio: guardar el } \\
\text { turno de palabra, escuchar, mirar al } \\
\text { interlocutor y mantener el tema }(2,8 \text {, } \\
5,6) \text {. } \\
\text { 4. Expresarse de forma oral mediante } \\
\text { textos que presenten de manera } \\
\text { organizada hechos y vivencias. }(2,1 \text {, } \\
3,5,6)\end{array}$ \\
\hline $\begin{array}{l}\text { 8. Utilizar la lectura como } \\
\text { fuente de placer y de } \\
\text { enriquecimiento personal, } \\
\text { como modo de apertura y } \\
\text { descubrimiento de otras } \\
\text { realidades y culturas y }\end{array}$ & $\begin{array}{l}\text { Bloque 3. Educación literaria } \\
5 \text {. Uso de los recursos de la } \\
\text { biblioteca de aula y de centro } \\
\text { incluyendo documentos audio- } \\
\text { visuales, como medio de } \\
\text { aproximación a la literatura y }\end{array}$ & $\begin{array}{l}\text { 5. Reproducir y representar textos } \\
\text { orales. }(2,1,5,6) \\
\text { 6. Conocer textos literarios de la } \\
\text { tradición oral y de la literatura infantil } \\
\text { adecuados al ciclo, así como algunos } \\
\text { aspectos formales simples de la }\end{array}$ \\
\hline
\end{tabular}




\begin{tabular}{|c|c|c|}
\hline $\begin{array}{l}\text { aproximarse a obras } \\
\text { relevantes de la tradición } \\
\text { literaria para desarrollar } \\
\text { hábitos de lectura. } 1,2,3,4 \text {, } \\
5,6,8 \text {. (Contenidos bloque } \\
\text { 3). }\end{array}$ & $\begin{array}{l}\text { como fuente de información y } \\
\text { de placer. } \\
\text { 6. Dramatización de } \\
\text { situaciones y de textos } \\
\text { literarios. }\end{array}$ & $\begin{array}{l}\text { narración y de la poesía con la } \\
\text { finalidad de apoyar la lectura de } \\
\text { dichos textos. }(2,4,5,6) \\
\text { 7. Iniciación al uso de la biblioteca del } \\
\text { aula y del centro, conocer su } \\
\text { funcionamiento y las posibilidades que } \\
\text { ofrece. }(2,7,5,6)\end{array}$ \\
\hline $\begin{array}{l}\text { 9. Iniciar la reflexión } \\
\text { progresiva sobre el uso de la } \\
\text { lengua, estableciendo } \\
\text { relaciones entre los aspectos } \\
\text { formales y los contextos e } \\
\text { intenciones comunicativas a } \\
\text { los que responden para } \\
\text { mejorar el uso personal del } \\
\text { lenguaje. } 1,2,5,6,8 \text {. } \\
\text { (Contenidos bloque } 4) \text {. }\end{array}$ & $\begin{array}{l}\text { Bloque 4. Conocimiento de la } \\
\text { lengua } \\
\text { 7. Observación de las } \\
\text { diferencias entre la lengua oral } \\
\text { y escrita a través de } \\
\text { experiencias de comunicación } \\
\text { en contextos diversos. } \\
\text { 8. Identificación de la palabra } \\
\text { como unidad básica con } \\
\text { significado y como } \\
\text { instrumento básico para la } \\
\text { segmentación de la escritura. }\end{array}$ & $\begin{array}{l}\text { 8. Comprender y utilizar la } \\
\text { terminología gramatical y lingüística } \\
\text { elemental, en las actividades } \\
\text { relacionadas con la producción y } \\
\text { comprensión de textos. }(2,5,6) \\
\text { 9. Reconocer, identificar y poner } \\
\text { ejemplos sencillos sobre las } \\
\text { principales profesiones y } \\
\text { responsabilidades que desempeñan las } \\
\text { personas del entorno. }(3,2,5,6) \\
\text { 10. Ordenar temporalmente algunos } \\
\text { hechos relevantes de la vida familiar o } \\
\text { del entorno próximo }(3,2,1,5,6) \\
11 . \text { Usar términos sencillos para } \\
\text { comentar las obras plásticas y } \\
\text { musicales observadas y escuchadas ( } 4 \text {, } \\
2,5,6) \\
12 \text {. Identificar diferentes formas de } \\
\text { representación del espacio y la } \\
\text { realidad }(4,2,1,5,6)\end{array}$ \\
\hline
\end{tabular}

Tabla 1. Ejemplo: Programación marco. Lengua castellana y literatura (primer ciclo)

Fuente: Elaboración propia

Como puede observarse, en cada una de las columnas se han relacionado los objetivos, contenidos y criterios de evaluación con los aspectos de las competencias seleccionados en el primer apartado. En los contenidos aparecen indirectamente, puesto que los objetivos se plantean para cada bloque con las competencias que se desarrollarán en cada uno. Lógicamente, pueden trabajarse de varias formas, en función de las actividades y metodología planteadas. Hay que recordar que, dado que se evalúan las competencias (y han de trabajarse todas) y el Decreto nos remite a los criterios de evaluación, tendremos que tomar criterios de todas y cada una de las áreas para el ciclo correspondiente. En este caso, se han tomado los criterios 1-2 del área de matemáticas; los criterios 3-8 del área de lengua castellana y literatura; los criterios 910 del área de conocimiento del medio; y los criterios 11-12 del área artística. Al lado de cada criterio se han destacado las competencias que pueden evaluarse con él, entre las que se incluyen las cuatro competencias no asociadas a un área afín y, por tanto, para las que no existen criterios de evaluación específicos. 


\section{Consecuencias curriculares de la enseñanza basada en competencias básicas}

La inclusión de competencias tiene una serie de consecuencias curriculares, pues de otro modo el cambio no sería tal. Todas las que exponemos las hemos extraído de la reflexión acerca del propio Decreto de Enseñanzas Mínimas, de nuestro planteamiento teórico y de conversaciones con diferentes grupos de trabajo con maestros. No obstante, algunas de ellas, se encuentran reflejadas, de una u otra forma, en parte de la bibliografía existente sobre el tema (Cfr. p. ej. Escamilla, 2008, 2009; MéndezGiménez, Sierra y Arizmendiarrieta y Mañana-Rodríguez, en prensa; Monereo, 2005; Toribio Briñas, 2010). En este apartado no se pretende agotar todas las posibles, pues pueden ser muchas, pero hemos tratado de sistematizarlas a partir de los diferentes elementos curriculares y la organización de los centros. El cambio en uno tiene su repercusión -como no puede ser de otra manera- en los demás. De forma resumida, y a modo de ejemplo, hemos querido mostrarlas en una lista abierta, que el lector podrá completar.

Teniendo en cuenta las competencias, los objetivos deben:

1. Exigir la conexión y trabajo conjunto entre las diferentes áreas.

2. Promover la adquisición del conocimiento en distintos contextos.

3. Favorecer el aprendizaje significativo y la metacognición.

4. Fomentar la autonomía del alumnado.

5. Facilitar la cooperación y relación entre el alumnado.

6. Impulsar la búsqueda de información en distintos soportes.

(...)

Teniendo en cuenta las competencias, los contenidos y actividades deben:

1. Plantearse de modo interdisciplinar.

2. Facilitar su transferencia a diferentes contextos.

3. Reforzar la comprensión significativa de los aprendizajes.

4. Conectar siempre con situaciones reales.

5. Fomentar el trabajo cooperativo del alumnado.

6. Emplear diferentes fuentes de información y favorecer el aprendizaje para una selección crítica de dichas fuentes.

(...)

Teniendo en cuenta las competencias, la metodología debe:

1. Reforzar la lectura y la escritura como una herramienta básica. 
2. Hacer más flexible la organización espacial y temporal en función de la interdisciplinariedad.

3. Realizar tareas en diversos contextos de aprendizaje.

4. Plantear situaciones-problema reales o simulados.

5. Utilizar metodologías cooperativas, de realización de proyectos, búsqueda de información, análisis y reflexión.

6. Emplear las TICs de forma crítica.

(...)

Teniendo en cuenta las competencias, la evaluación debe:

1. Tener en cuenta conocimientos, procesos y adaptación a contextos.

2. Utilizar instrumentos variados: observación, producciones diversas (individuales y en grupo), pruebas orales y escritas.

3. Continuar con una equilibrada combinación de evaluación formativa y sumativa.

4. Promover la autoevaluación como forma de aprender a aprender y facilitar la autonomía personal.

5. Comprobar la articulación de conocimientos teóricos y prácticos.

6. Valorar de forma prioritaria la resolución de situaciones-problema, las respuestas creativas adaptadas a diferentes contextos y la búsqueda selectiva de información.

(...)

La inclusión de competencias en el currículo exige algunos cambios en los centros:

1. Reestructurar las programaciones. Se trata de un cambio de enfoque, no de incluir un apartado más.

2. Crear en cada centro grupos cooperativos de trabajo docente interdisciplinar.

3. Reajustar horarios que permitan la inclusión de los cambios.

4. Incluir en las reuniones de evaluación el desarrollo de las competencias básicas, además de las diferentes materias.

5. Realizar planes de formación docente para la mejora de la enseñanza y desarrollo de las competencias básicas, según las necesidades.

6. Establecer redes de colaboración docente entre centros para compartir experiencias y resolver conflictos.

$(\ldots)$ 


\section{Hacia un planteamiento metodológico interdisciplinar}

La inclusión de las competencias tiene repercusión, como hemos visto, en los diferentes elementos del currículo y, por consiguiente, exige una nueva forma de plantear las programaciones. Con el tiempo, según se ha indicado páginas atrás, sería deseable que también provocara un cambio en la organización de los espacios y tiempos en los centros escolares. Pero, entre todos los cambios, el que parece más importante es el metodológico, atendiendo a dos vertientes que se encuentran en íntima interrelación:

1. La metodología de aula, de manera que las actividades estén interrelacionadas, sirviendo de conexión entre las diferentes materias. Esto se hace imprescindible por el propio concepto de competencia. Ferreiro destaca que "el concepto de competencia y el del enfoque basado en competencia, son por su naturaleza, constructos integradores, holísticos" (Ferreiro Gravié, 2011, p. 12). Las competencias se ejercitan y desarrollan resolviendo problemas en diversas situaciones, lo que hace imposible la separación entre las distintas disciplinas y contenidos. Habrían de establecerse puentes y actividades interdisciplinares que evitaran la desconexión entre distintos contenidos como si fueran compartimentos estancos. En este sentido, es especialmente sugerente el concepto de "actividad rica", que fue propuesto, desde la investigación en Didáctica de las Matemáticas, por Broomes (1989). Entre las características que este autor le atribuye a una "actividad rica" destacamos las siguientes: permite establecer conexiones entre distintas áreas del currículo, sirve como introducción y motivación para un contenido básico, supone un reto para el alumnado y permite diferentes ritmos de aprendizaje, facilita la implicación de los estudiantes, es flexible, y pretende no sólo que se busquen respuestas, sino generar buenas preguntas (Llach Carles y Alsina i Pastells, 2009). Sin detenernos a establecer semejanzas y diferencias, hay que recordar que Raths (1971) -aunque con un objetivo diferente- ya había propuesto años antes unos criterios para la selección de actividades que, en parte, coinciden con algunas de las características señaladas por Broomes.

Como facilitador, el docente no sólo tendrá que presentar los contenidos, sino conducir a los discentes a través de diferentes caminos que comuniquen unos contenidos con otros, recorriendo distintos trayectos que permitan una visión más amplia y profunda del mapa de contenidos, al modo de una estructura hipertextual. La inclusión de las competencias exige un planteamiento diferente de los procesos de enseñanza-aprendizaje, orientando la "práctica docente hacia esa concepción integrada, aplicada y contextualizada del aprendizaje (...) buscando la interrelación de conocimientos, la movilización de los saberes, la reflexión crítica sobre lo aprendido, la apertura de nuevos caminos hacia el saber" (Tiana Ferrer, 2011, p. 72). Este cambio en la concepción de los procesos de enseñanza-aprendizaje ha de fomentar una mayor interdisciplinariedad. Fernández Ríos destaca que el aprendizaje interdisciplinar promueve tanto en estudiantes como en profesores "la capacidad para reconocer, evaluar y poner en práctica perspectivas teóricometodológicas múltiples para solucionar mejor los problemas" (Fernández Ríos, 2010 , p. 161). Aunque él se refiere primordialmente al aprendizaje universitario, no 
hay que olvidar que los estilos de aprendizaje (por supuesto, adaptando los niveles de dificultad) hay que desarrollarlos desde la etapa escolar para que den mejor fruto en la etapa universitaria. Es interesante tener en cuenta la propuesta de Morín para la educación del futuro, en la que apuesta por un cambio en la forma de conocimiento, dejando de lado las informaciones o datos aislados, para tener en cuenta el contexto, lo global (relación entre el todo $\mathrm{y}$ las partes), lo multidimensional y lo complejo (Morín, 2001).

Ferreiro destaca la necesidad de coherencia interna entre una teoría, la metodología empleada y el método aplicado, añadiendo que para poder realizar un trabajo por competencias en el aula, se hace preciso como condición necesaria las competencias profesionales del maestro (Ferreiro Gravié, 2011, pp. 12-13). De acuerdo con su afirmación, podría concluirse que para poder cambiar la metodología de aula, sería necesario también una transformación en la metodología docente fuera del aula, que es la que facilitará y constituirá el sustrato imprescindible para que fructifiquen los cambios planteados.

2. La metodología fuera del aula es tanto la fase previa como posterior a las clases. Si quieren ser capaces de abordar unas actividades interdisciplinares que faciliten el desarrollo de las competencias en el alumnado, los docentes necesitarán, cada vez más, trabajar de modo cooperativo con otros docentes -sean tutores o especialistas. Los docentes tendrán que empezar a colaborar estrechamente y fomentar el trabajo en equipo. Rodríguez Gómez (2009) destaca el trabajo en equipo, para abordar tareas de mayor complejidad y procurar resultados creativos, como un aspecto muy importante de la competencia docente, fomentando una cultura colaborativa. Ya años antes, Perrenoud (2004) incluía el trabajo en equipo dentro de lo que el llama 'competencias de referencia' para el profesorado del siglo XXI.

Este planteamiento exigiría una mayor comunicación entre docentes de un mismo curso, ciclo y etapa, y la creación de un seminario permanente como plataforma de información e intercambio de actividades y contenidos. Así, para una buena práctica de la enseñanza-aprendizaje de competencias se habrían de crear grupos de trabajo docente en los que participen todos los implicados en un mismo curso, e incluso mejor en el mismo ciclo. Sólo de esta forma se puede lograr una perspectiva más unitaria y significativa en el aprendizaje y, asimismo, allanar el camino de la práctica docente con la experiencia y saber que aportan los demás participantes en el grupo. La creación de dichos grupos de trabajo exige necesariamente una reorganización del tiempo y, como sugieren Méndez-Giménez, López-Téllez y Sierra y Arizmendiarrieta (2009, p. 56), quizá cierto cambio estructural en la organización de los centros escolares. Ello no carece de complejidad. No obstante, la propuesta del desarrollo de competencias básicas desde una perspectiva interdisciplinar así lo exige -tanto en la práctica, como también en el planteamiento teórico- si realmente se quiere mejorar la enseñanza y el aprendizaje, y no sólo llamar de otra manera a la práctica de siempre. 
No es posible trabajar las ocho competencias básicas, con la descripción que aparece de cada una de ellas en el Anexo I del Real Decreto de Enseñanzas Mínimas (de Primaria y ESO), sin una coordinación entre las áreas, así como intraciclos e interciclos. Para que los cambios educativos sean realmente eficaces y constituyan una mejora no basta, por tanto, con un cambio de nomenclatura y un gran esfuerzo burocrático, sino que han de llevar consigo un cambio de enfoque y una gran apuesta e inversión de tiempo y esfuerzo en formación. Partimos del convencimiento de que, aunque la preparación de los tutores y los diferentes especialistas fuera la mejor posible, resultaría muy complejo trabajar todas las competencias con la misma intensidad. Por ello, parece una necesidad intrínseca a la enseñanza-aprendizaje de competencias básicas que el trabajo de los docentes sea cooperativo e interdisciplinar, de forma que puedan apoyarse unos a otros con diferentes propuestas de actividades y tareas que permitan trabajar a través de cada contenido todas las competencias.

La propuesta parece lógica si se quiere cambiar, como así parece, el modelo tradicional de los procesos de enseñanza-aprendizaje. Pero para reformar la práctica docente, y añadir nuevas competencias profesionales, es necesario tener en cuenta algunas dificultades con las que los docentes se encuentran. Monereo (2010) realiza una revisión de un conjunto de investigaciones, que analizan su resistencia al cambio, explicando: factores de tipo personal-emocional, como la falta de seguridad para desarrollar una nueva competencia; factores profesionales, que aluden a la desmotivación ocasionada por distintas reformas en las que no tienen capacidad de decisión; y factores institucionales, que básicamente no facilitan los medios para la construcción de una cultura colaborativa. Todo ello requiere de propuestas de formación que logren paliar dichos problemas, así como un mayor apoyo institucional.

\section{Conclusión}

La introducción de las competencias en el curriculum no puede consistir en la inclusión de un apartado más, sin que afecte al resto de componentes más que de modo superficial. Exige, por el contrario, un replanteamiento de los componentes curriculares en torno a las mismas, de forma que las competencias sean el núcleo o eje en torno al cual se planteen objetivos, contenidos, actividades, metodología y evaluación, sirviendo como nexo de unión entre todos ellos. El cambio de modelo supone, por tanto, una modificación sustancial en la forma de entender los procesos de enseñanzaaprendizaje. La necesidad de una nueva reestructuración de las programaciones trae consigo consecuencias y exigencias para todos los elementos del curriculum, así como para las condiciones organizativas que lo hagan posible. Así, la estructura del centro escolar habría de estar al servicio de un planteamiento didáctico realmente sistémico en el que la interdisciplinariedad sería el medio idóneo para el equilibrio del sistema escolar. Dicha interdisciplinariedad constituye el reto para la actual enseñanza a través del desarrollo de competencias. 
Tanto desde el punto de vista teórico (en el diseño) como práctico (en su desarrollo), la pregunta inmediata y más urgente recae sobre el 'cómo', es decir, sobre la metodología necesaria para poder lograr la innovación requerida. En estas páginas se ha atendido a dos frentes: la metodología de aula y la metodología fuera del aula, como condición de posibilidad de la primera.

a) Respecto a la primera, la presencia de las competencias como elemento integrador en las nuevas programaciones, exige una mayor apertura y flexibilidad en el diseño y desarrollo curricular, abriendo las compuertas entre disciplinas que hasta ahora han sido entendidas de modo cerrado e independiente. Para lograrlo, cobra especial importancia el papel que desempeñan las tareas como nicho ecológico en el que las competencias pueden ejercitarse. Dichas tareas no han de plantearse como ejercicios o actividades al modo tradicional, sino como zonas de intersección entre disciplinas, como puentes que faciliten al alumnado darse cuenta de la conexión intrínseca entre los diferentes contenidos. Esta reforma metodológica tiene, lógicamente, algunas repercusiones en los dos agentes del proceso. Sólo indicaremos brevemente una de ellas, vista desde ambos lados de la relación didáctica:

-Propicia un cambio en el rol docente que, ahora tendrá que actuar menos como transmisor que como facilitador. Ello le exigirá un mayor esfuerzo de preparación de tareas e integración de aprendizajes, además de tener que vencer la resistencia a los cambios tanto en la planificación curricular como en la práctica.

-Promueve, de modo necesario, una mayor actividad en el alumnado, pues es la única forma que tiene de incorporarse en el proceso de enseñanza-aprendizaje. Con una metodología basada en la transmisión, los estudiantes pueden implicarse más o menos, e incluso pueden descolgarse del proceso sin poder, a veces, percibirlo hasta que es tarde. Sin embargo, la implicación que supone el desarrollo de competencias, a través de diversas tareas, facilita que el aprendizaje sea más significativo $\mathrm{y}$, por tanto, la motivación mayor al advertir un avance en el conocimiento.

b) Respecto a la segunda, urge reflexionar y profundizar en un cambio en el modo de trabajar más allá de la metodología empleada en el aula. Ésta, para tener éxito -dada su complejidad- necesita el soporte formativo, personal e institucional que haga posible el profundo cambio metodológico (de aula) aludido. La formación individual es necesaria para la adquisición de nuevas competencias profesionales, pero ésta se queda coja sin un planteamiento institucional que no sólo permita, sino que fomente una mayor comunicación profesional docente. El cambio ha de ir orientado hacia la creación de equipos docentes en los que, mediante el trabajo cooperativo entre el profesorado de las mismas y diferentes áreas, se facilite la propuesta de actividades y tareas interdisciplinares que ayuden a desarrollar todas las competencias de forma integrada. La transmisión y comunicación mutua de experiencia y experiencias puede redundar en una mayor implicación en la tarea, lo que facilitará necesariamente un aumento de la motivación y una mejora en la calidad docente. Así, este planteamiento cooperativo e interdisciplinar en el trabajo docente no se propone sólo como una 
metodología para facilitar el desarrollo de las competencias en el aula, sino que, dado su carácter relacional de apoyo e intercambio, pretende ser, en sí mismo, formativo.

\section{Referencias bibliográficas}

ARREAZA BEBERIDE, F. (2009). La clase y el aula son escenarios insuperables para movilizar los conocimientos. Avances en Supervisión Educativa, 10, 1-10.

BROOMES, D. (1989). Using goals to construct useful forms of school mathematics. En Keitel, C, Damerow, P., Bishop, A.J. y Gerdes, P. (eds.). Mathematics, Education and Society. Paris: UNESCO, Col. Science and Tecnology Education, Document Series $n^{\circ} 35$.

CLIMENT BONILLA, J.B. (2010). Reflexiones sobre la Educación Basada en Competencias. Revista Complutense de Educación, 21(1), 91-106.

DECRETO 56/2007, de 24 de mayo, por el que se regula la ordenación y establece el currículo de la Educación primaria en el Principado de Asturias (B.O.P.A. n 140 , sábado 16 de junio de 2007).

DOMINGO SEGOVIA, J. y BARRERO FERNÁNDEZ, B. (2010). Competencias básicas y aprendizajes imprescindibles. En Moral Santaella, C. (Coord.). Didáctica. Teoría y práctica de la enseñanza. Madrid: Pirámide.

ESCAMILlA, A. (2008). Las competencias básicas. Claves y propuestas para su desarrollo en los centros. Barcelona: Graó.

ESCAMILLA, A. (2009). Las competencias en la programación de aula (Infantil y Primaria (3-12 años). Barcelona: Graó.

FEITO ALONSO, R. (2010). De las competencias básicas al curriculum intregrado. Revista Qurriculum, 23, 55-79.

FERNÁNDEZ-RÍOS, L. (2010). Interdisciplinariedad en la construcción del conocimiento: ¿Más allá de Bolonia? Innovación educativa, 20, 157-166.

FERREIRO GRAVIÉ, R.F. (2011). Tres vértices del triángulo de las Competencias Didácticas: Teoría, Metodología y Método. Revista Complutense de Educación, 22 (1), 11-23.

GARCÍA PEINADO, B. (2009). Cómo incluir las competencias en la programación didáctica. Teoría del entrenamiento básico. Avances en Supervisión Educativa, 10, 1-15.

JURADO VALENCIA, F. (2009). El enfoque sobre competencias: Una perspectiva crítica para la educación. Revista Complutense de Educación, 20 (2), pp. 343-354. 
LLACH CARLES, S. y ALSINA I PASTELLS, A. (2009). La adquisición de competencias básicas en Educación Primaria: una aproximación interdisciplinar desde la Didáctica de la Lengua y de las Matemáticas. Revista Electrónica Interuniversitaria de Formación del Profesorado, 12 (3), 71-85.

MARCO STIEFEL, B. Competencias básicas. Hacia un nuevo paradigma educativo. Madrid: Narcea.

MÉNDEZ-GIMÉNEZ, A., SIERRA Y ARIZMENDIARRIETA, B. y MAÑANARODRÍGUEZ, J. (en prensa). Percepciones y creencias de los docentes de primaria del Principado de Asturias sobre las competencias básicas, Revista de Educación.

MÉNDEZ-GIMÉNEZ, A., LÓPEZ-TÉLLEZ, G., Y SIERRA, B. (2009). Competencias básicas: sobre la exclusión de la competencia motriz y las aportaciones desde la Educación Física. Retos: Nuevas tendencias en Educación Física, Deporte y Recreación, 16, 51-57.

MONEREO, C. (Coord.) (2005). Internet y competencias básicas. Barcelona: Graó.

MONEREO, C. y POZO, J.I. (2007). Competencias para (con)vivir con el siglo XXI. En MONEREO, C. y POZO, J.I. (Coords.) Monográfico sobre competencias básicas. Cuadernos de Pedagogía, 370, 12-17.

MONEREO, C. (2009). La autenticidad de la evaluación. En CASTELLÓ, M. (Coord.) La evaluación auténtica en enseñanza secundaria y universitaria. Barcelona: Edebé.

MONEREO, C. (2010). ¡Saquen el libro de texto! Resistencia, obstáculos y alternativas en la formación de los docentes para el cambio educativo. Revista de Educación, 352, 583-597.

MORÍN, E. (2001). Los siete saberes necesarios para la educación del futuro. Barcelona: Paidós.

MOYA OTERO, J. (2008). Las competencias básicas en el diseño y el desarrollo del currículo. Revista Qurriculum, 21, 57-78.

OLIVEROS MARTÍN-VARÉS, L. (2006). Identificación de competencias: una estrategia para la formación en el Espacio Europeo de Educación Superior. Revista Complutense de Educación, 17 (1), 101-118.

ORDEN HOZ, A. de la (2011). El problema de las competencias en la educación general. Bordón. Revista de Pedagogía, 63, 47-61.

PERRENAUD, P.H. (2004). Diez nuevas competencias para enseñar. Barcelona: Graó.

RATHS, J.D. (1971). Teaching Without Specific Objectives. Educational Leadership, 714-720. 
REAL DECRETO 1513/2006, de 7 de diciembre, por el que se establecen las enseñanzas mínimas de la Educación Primaria (B.O.E. núm. 293, viernes 8 de diciembre de 2006).

REAL DECRETO 1631/2006, de 29 de diciembre, por el que se establecen las enseñanzas mínimas de la Educación Secundaria Obligatoria (B.O.E. núm. 5, viernes 5 de enero de 2007).

RIBES IÑESTA, E. (2011). El concepto de competencia: su pertinencia en el desarrollo psicológico y la educación. Bordón. Revista de Pedagogía, 63, 33-45.

RODRÍGUEZ GÓMEZ, J.M. (2009). De la noción de calificación a la noción de competencia: sugerencias para el profesorado. Revista Electrónica Interuniversitaria de Formación del Profesorado, 12(2), 93-101.

RODRÍGUEZ TORRES, J. (2010). De las programaciones didácticas a la unidad didáctica: incorporación de competencias básicas y la concreción de tareas. Revista Docencia e Investigación, 20, 245-270.

ROSALES LÓPEZ, C. (2010). La planificación de la enseñanza por competencias: ¿Qué tipo de innovación implica? Innovación educativa, 20, 77-88.

SIERRA Y ARIZMENDIARRIETA, B., MÉNDEZ-GIMÉNEZ, A. y MAÑANARODRÍGUEZ, J. (2012). Necesidad y propuesta de un procedimiento para programar por competencias básicas, Aula Abierta, 40(3).

TIANA FERRER, A. (2011). Análisis de las competencias básicas como núcleo curricular en la educación obligatoria española. Bordón. Revista de Pedagogía, 63, 63-75.

TORIBIO BRIÑAS, L. (2010). Las competencias básicas: el nuevo paradigma curricular en Europa. Foro de Educación, 12, 25-44.

VISO ALONSO, J.R. (2010). Qué son las competencias. Vol. 1. Madrid: EOS.

ZABALA, A. y ARNAU, L. (2007). 11 ideas clave. Cómo aprender y enseñar competencias. Barcelona: Graó. 


\section{Correspondencia con los autores}

Beatriz SIERRA Y ARIZMENDIARRIETA

Departamento de Ciencias de la Educación

Facultad de Formación del Profesorado y Educación, C/Aniceto Sela s/n

Universidad de Oviedo (33005-Oviedo)

Correo-e: bsierra@uniovi.es

Antonio MÉNDEZ-GIMÉNEZ

Departamento de Ciencias de la Educación

Facultad de Formación del Profesorado y Educación, C/Aniceto Sela s/n

Universidad de Oviedo (33005-Oviedo)

Correo-e: mendezantonio@uniovi.es

Jorge MAÑANA-RODRÍGUEZ

Centro de Ciencias Sociales y Humanas (CCHS),

Consejo Superior de Investigaciones Científicas (CSIC).

C/ Albasanz 26-28 (Madrid)

Correo-e: jorge.mannana@cchs.csic.es 